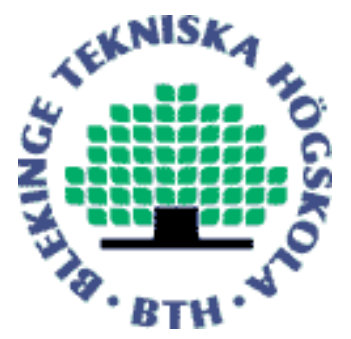

Copyright (C) 2012 IEEE.

Citation for the published paper:

Mobile video sensitivity to packet loss and packet delay variation in terms of QoE

Tahir Nawaz Minhas, Oziel Gonzales Lagunas, Patrik Arlos, Markus Fiedler

19th International Packet Video Workshop (PV)

2012 Munich, Germany

This material is posted here with permission of the IEEE. Such permission of the IEEE does not in any way imply IEEE endorsement of any of BTH's products or services Internal or personal use of this material is permitted. However, permission to reprint/republish this material for advertising or promotional purposes or for creating new collective works for resale or redistribution must be obtained from the IEEE by sending a blank email message to pubs-permissions@iee.org.

By choosing to view this document, you agree to all provisions of the copyright laws protecting it. 


\title{
Mobile Video Sensitivity to Packet Loss and Packet Delay Variation in Terms of QoE
}

\author{
Tahir Nawaz Minhas*, Oziel Gonzalez Lagunas ${ }^{\dagger}$, Patrik Arlos*, Markus Fiedler* \\ ${ }^{*}$ School of Computing \\ Blekinge Institute of Technology \\ Email: \{Tahir.Nawaz.Minhas, Patrik.Arlos, Markus.Fiedler\}@bth.se \\ ${ }^{\dagger}$ Student of \\ Blekinge Institute of Technology \\ Email:ozielglez@gmail.com
}

\begin{abstract}
With the growth of the mobile internet, the popularity of multimedia services and applications have increased rapidly. As a result, end-users become quality-conscious. To fulfill the users' expectations, the study of quality of experience (QoE) is becoming very important for both researchers and service providers. This paper analyses the impact on perceived quality of received videos encoded with the $\mathbf{H . 2 6 4}$ baseline profile, which is suitable for mobile video, and streamed through an emulated network with packet loss and packet delay variation. To evaluate the video QoE, tests are conducted on a mobile device and on a laptop. The users' responses show that the baseline profile of H.264 is very sensitive to packet loss and packet delay variation. Moreover, there is no considerable impact on users' perception either test is conducted on the mobile device or on the laptop by playing the same resolution video.
\end{abstract}

\section{INTRODUCTION}

The use of applications on mobile phones and particularly on smart phones are increasing with rapidly growing mobile internet. Amongst other services, the popularity of multimedia services and applications are increasing rapidly. Moreover, the capabilities of mobile phones in terms of processing power, memory, color display and other functionalities are improving continuously. As a result of this growth and exposure, the user expects good Quality of Experience (QoE). Real-time applications and multimedia services can affect user perception very quickly, as compared to other applications on Internet. Due to this consciousness of the users, service providers, application developers and research community are focusing on user QoE. Therefore, to send the video across the network, several techniques for video compressions are in use [1], one of the most widely used codecs is H.264/AVC (Advanced Video Coding). It is used with different applications and each has its own specific requirement, so it is divided into different profiles with respect to their usage [2]. For example, the mobile applications use the Baseline Profile (BP) of H.264.

Nowadays the mobile phones are used for many tasks beyond making phone calls [3]. Especially mobile videos are getting popular. Mobile video quality can be degraded over the internet due to network disturbances, such as packet loss and packet delay variation [4].

In this paper we study the QoE of H.264 baseline profile for defined values of packet loss and delay variation, which is suitable for mobile video streaming [2]. Moreover, we also investigate the role of video playing device (in this case mobile phone and laptop) on QoE for a fixed resolution video. In order to achieve these research objectives, an experimental set-up was implemented to emulate the characteristics of a network, over which videos were transmitted. Subjective assessments were realised following the recommendations from the International Telecommunications Union (ITU) [5], [6]. The results are calculated and presented using Mean Opinion Score (MOS) and statistical methods.

The remainder of this paper is organized as follows. Section II covers the related work and Section III describes the experiment setup and assessment methodology. The results are presented and discussed in Section IV, while Section V concludes the paper.

\section{RELATED WORK}

A set of studies have addressed the video quality perception for video and different codecs. Calyam et al. [7] made a comparative study of subjective and objective video quality. They added disturbances of loss, delay and jitter to the same video sequence and found that jitter has the biggest effect. Claypool et al. [8] realised a subjective perception study for the codec MPEG-1 making variations of jitter and packet loss. Hands and Wilkins [9] performed video quality assessment by using the codec MPEG-1 and changing the packet loss and burst size.

Previous work has also addressed studies employing the codec H.264, using subjective and objective methods. Jusmisko et al. [10] worked in a subjective analysis comparing different codecs H.263, H.264 and XviD for mobile devices. Lin et al. [11] presented a model for packet prioritization for different GOP structures of H.264 and MPEG-2. The article by Loguinov and Radha [12] analysed the behaviour of different parameters in video coded with MPEG-4. De Simone et al. [13] offer a database of H.264 videos for research community and made experiments with packet loss.

Some studies that have addressed the quality of video for H.264, mention the profile used. For example, [11] and [13] used the high profile, [14] used the main profile, and Ries et 
al. [15] used the baseline profile for quality estimation based in motion characteristics.

Furthermore, studies of video quality perception for mobile devices in some cases are carried out using a monitor or big LCD display instead of a mobile phone [16], [17]. Further, in the study done by Jumisko et al. [10], mobile phones were used but the phones were attached to a fixed stand, so the user was not able to manipulate the phone as it would be the case in real life scenarios.

People have different perceptions of quality for different media devices. Television and computers are usually at a fixed distance in contrast with hand-held devices on which distance and angle can be adjusted easily by the user. The perception changes according to the image size. Previous TV experiments carried out found that the general rule to be the bigger the image the better quality perceived [18].

Consequently, a study that tests the user perception of video quality on different network conditions, for packet loss and delay variation encoded with H.264 and considers characteristics for mobile devices, will contribute to understanding the expectations of the users with mobile devices. Further, an understanding of the role the device plays in the user perception of packet loss and delay variation is required.

\section{EXPERIMENT SETUP}

The emulation setup shown in Figure 1 is used to carry out the experiments. The Streamer (S) is responsible to send the original encoded video sequences through the Shaper (TS) to the Player (P), using the UDP protocol. A full-duplex link of bandwidth $100 \mathrm{Mbps}$ is used from $\mathrm{S}$ to $\mathrm{D}$. For streaming, VLC is used, while for delay variation and packet loss shaping the Network Emulator (NetEm) is used, as it provides the best delay shaping as compared to other shapers [19].

Computer $\mathrm{S}$ is a Toshiba laptop with an Intel Core Duo T2450 2.00GHz processor architecture running Ubuntu 10.04 LTS Linux, while computer $\mathrm{P}$ is a MacBook Pro with an Intel CPU Core 2 Duo $2.40 \mathrm{GHz}$, running MacOS X 10.5. Both computers are running the VLC media player version 1.1.3. The shaper computer has an Intel CPU $2.66 \mathrm{GHz}$ with Linux kernel 2.6.23.9. Since we are using a Linux kernel version higher than 2.6.14, NetEm performs re-ordering of packets if the jitter exceeds the inter-packet time and if the default configuration is used. Thus, in our experiments, we may face packets re-ordering, which is not corrected. We saved the streamed and disturbed videos in database (DB) using VLC. The jitter buffer of VLC is set to be zero to see the immediate effect of network impairments. In the second step, the (impaired) videos will be played back as shown in Figure 2, which be explained in detail at the end of this section.

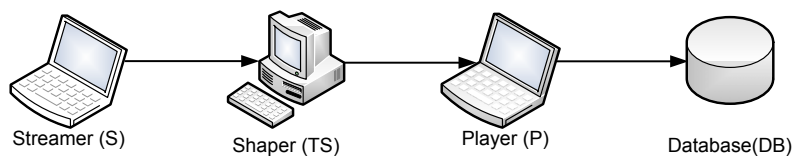

Fig. 1. Experiment Setup, Part 1: Disturbing and recording.

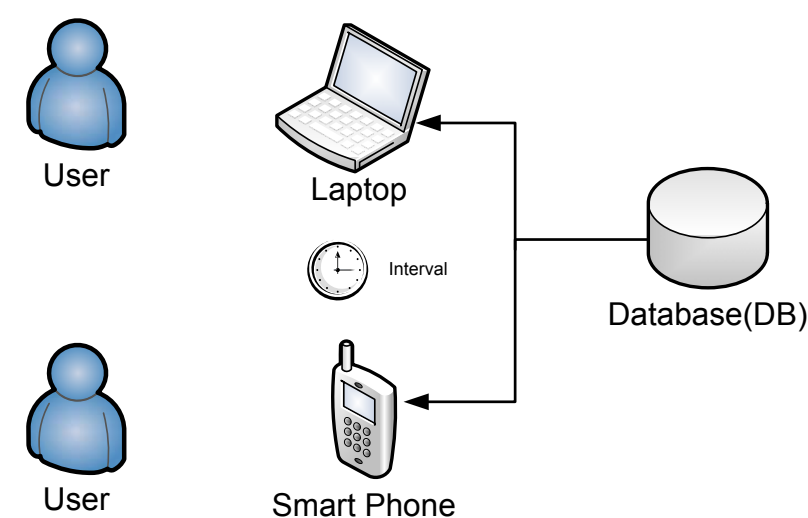

Fig. 2. Experiment Setup, Part 2: Playback and user ratings.

The shaping values were chosen from literature review and laboratory testing. Laboratory tests were performed for all experimental values of packet loss or delay and for the final assessment; we selected the values where the MOS ratings were changed significantly. This happens for instance when delays of $200 \mathrm{~ms}$ or more are applied, due to glitches at the start-up of the video that affected the user rankings. For this reason, we applied a fixed delay of $150 \mathrm{~ms}$. In case of delay variation $(\Delta D)$, a test was performed for $0 \mathrm{~ms}$ to $50 \mathrm{~ms}$ with an increment of $2 \mathrm{~ms}$. We found that the video content got lost when $\Delta D>16 \mathrm{~ms}$. So the setting used for the shaping of delay $(D)$ and varying delay $(\Delta D)$ were 150 $\pm\{0,2,4,8,12,16\} \mathrm{ms}$ with normal distribution. Similarly, for packet loss shaping experiments were performed for $0.1 \%$ to $1 \%$ with increment of $0.1 \%$ and from $1 \%$ to $15 \%$ with the increment of $1 \%$. Various studies modelling packet loss in different networks show values below $10-15 \%$ [4], [9]. In [20], the authors consider loss ratios between 0 to $90 \%$ while in [21] utility value of videos were estimated for $1 \%$ to $10 \%$ of packet loss. In laboratory tests, all video sequences are shown to the users, and those values of packet loss were selected that caused significant changes in MOS. In particular, we found that user rating remained similar from $0.1 \%$ to $0.4 \%, 0.5 \%$ to $0.8 \%$ and so on. In case of packet loss greater than $7 \%$ the content of video was lost. Thus, the selected values for packet loss became $0.4 \%, 0.8 \%, 3 \%, 5 \%$ and $7 \%$.

Video quality assessments can be objective and subjective. In subjective quality assessment, the description of quality is based on human perception and can be different between subjects [7]. Human perception involves various aspects of human psychology and viewing conditions, such as vision ability; lighting conditions; preference for content; displaying devices; and understanding of the rating criteria [22]. Absolute Category Rating (ACR) is considered to be well-suited for qualification tests [5] and was applied in several studies [23], [14], [13].

Another option considered was the software called Perceptual Evaluation Video Quality (PEVQ) [24]. It is a software approved by the VQEG and ITU-T that performs an objective analysis of the video and gives a good correlation with the 
subjective MOS. However, in [25] the authors questioned the performance of PEVQ for the particular case of freezing and jumping artifacts of video as compared to the user's rating.

The videos were taken from a commonly used repository for the purpose of video quality assessment studies [26] as suggested by De Simone et al. [13]. The selected videos used for experiments are Football, Foreman and Hall-monitor. The length of each video sequences is between eight and ten seconds, which is the length suggested by ITU-T [5].

The video sequences used for this study are encoded with H.264 Baseline Profile, level 1.3 because it is recommended for mobile videos. We used FFMPEG with libx264 for encoding. Each sequence has a frame rate of $30 \mathrm{fps}$ and a bitrate of $768 \mathrm{kbps}$. Previous video studies for mobile have used the QCIF $(176 \times 144)$ resolution [10], [15], [16]; in some cases using mobile devices and some others displaying the video on a computer monitor displaying the QCIF resolution. For this study was decided to use higher resolution Quarter Video Graphics Array $(\mathrm{QVGA})(320 \times 240)$ to cover the new segment of modern phones, which also should be capable of consuming 768 kbps.

Assessment Methodology: The assessment sessions were held in rooms conforming to the specifications of the ITU$\mathrm{T}$ [5] at the premises of two academic institutions in Kristianstad and Karlskrona in Sweden.

Two mobile devices were used for the assessment. A laptop Toshiba Satellite with an Intel Core Duo T2450 2.00GHz with a LCD display of 15.4 inches (diagonal) widescreen TruBrite TFT with resolution $1280 \times 800$, and an iPhone $3 \mathrm{G}$. The media player VLC version 1.1.3 were used on the laptop and the standard media player from Apple Inc. on the iPhone Mobile Phone. In some cases, the series of experiments started on the laptop, in other cases on the mobile. The impaired videos were shown in random order. There was a short break between the series on both devices in order to make the viewers relax.

For the mobile phone the user was able to choose the angle and distance to watch the videos. This imitates what happens in a real-world environment where the user can move the device as desired to achieve a comfortable viewing position.

The video sequences shown on the laptop complied with the recommendations made by the ITU-T [5]. The videos were played in VLC using the original resolution of the video sequence $(320 \times 240)$, in the center of the screen, with the background set to fifty percent grey.

\section{REsults}

This section presents the results collected from the assessment sessions. During the assessment sessions, 34 volunteers graded the 33 videos sequences using the scale Excellent (5), Good (4), Fair (3), Poor (2) and Bad (1). Each video was shown on both a laptop and on a mobile phone. In total 66 video sequences were shown to the subjects.

\section{A. Packet loss}

Figures 3 and 4 show the graphs of the MOS for the packet loss for the laptop and the mobile phone, respectively. The graphs illustrate that the smaller the values of loss the higher MOS, indicating better user perception of the video. As packet loss increases, the MOS drops steeply, but it remains "fair" until values of packet loss between $0.4 \%$ and $0.8 \%$. From $5 \%$ packet loss the value of the MOS is close to 1 (bad). This behaviour suggests that packet loss is an important factor to consider. If videos with several percent of loss were provided as a service, users might consider stop watching.

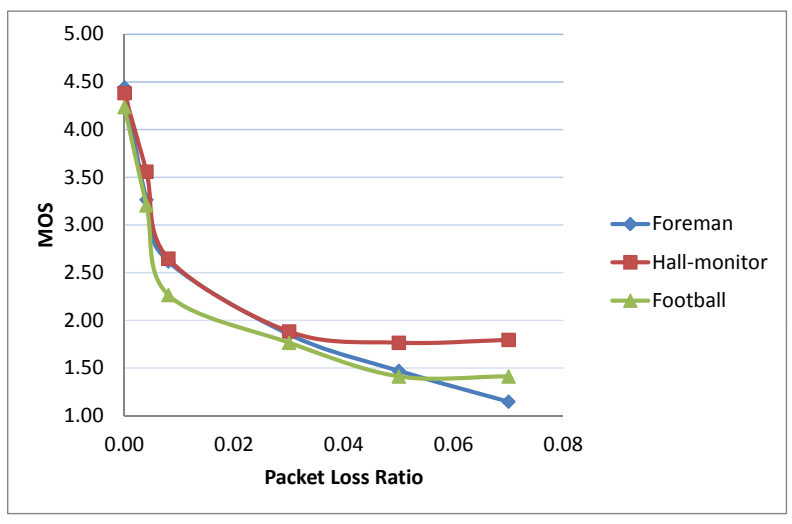

Fig. 3. MOS for packet loss displayed on the laptop

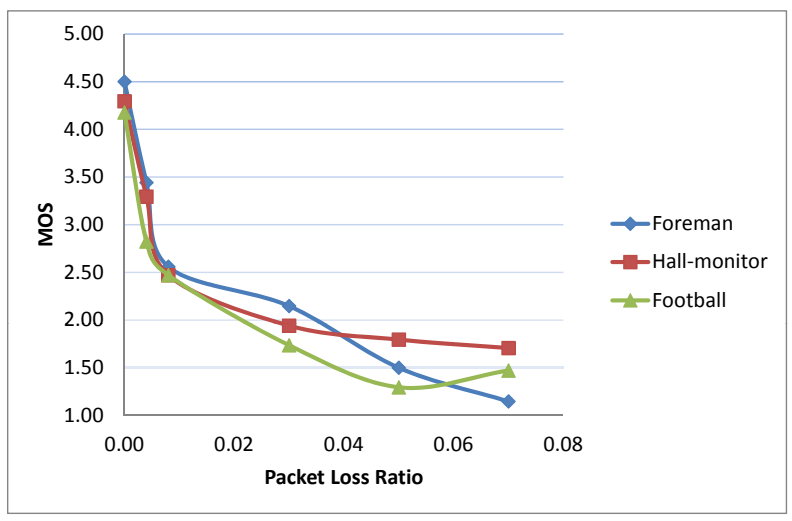

Fig. 4. MOS for packet loss displayed on the mobile phone

The video sequence encoded with H.264 and without distortions was graded with a MOS that lies between 4.50 and 4.18, although there are no disturbances. This shows that the viewers in some cases are reluctant to score the videos as "excellent." This behaviour was also found in other studies [14].

For the different videos affected by packet loss, it is noticed that the video with the lowest MOS, in both cases, is the Football video. For the video Hall-monitor in both devices the MOS decline to poor (2), and then it seems that there is not a big variation between $3 \%$ and $7 \%$. The video sequence Foreman is the video with the lowest MOS for $7 \%$ loss.

The values calculated for the confidence interval for packet loss are shown in Figure 5. The $\mathrm{x}$-axis shows the video sequence $(\mathrm{FM}=$ Foreman, $\mathrm{HM}=$ Hall-monitor, $\mathrm{FT}=$ Football $)$, the device that was used to present the video sequence to the observer ( $\mathrm{L}=$ Laptop; $\mathrm{P}=$ Mobile phone) and the percentage 
of packet loss emulated. The y-axis refers to the MOS. We can see that there is not a big difference within the three different video sequences for each of the packet loss values. The tendency shows that for bigger values of packet loss, the rate degrades for all the video sequences. In general, for values above $5 \%$ of packet loss the observers rate close to 1 (bad), with slightly higher MOS values in case of the Hall-monitor video sequence in both devices.

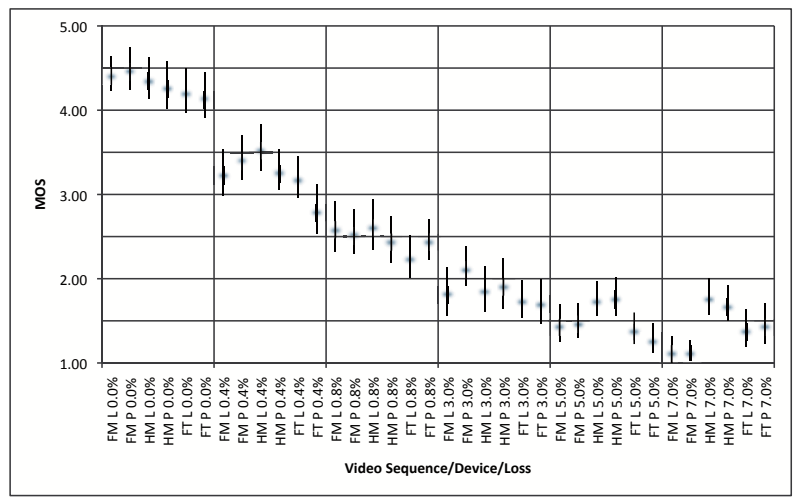

Fig. 5. 95\%-Confidence interval for packet loss

\section{B. Packet Delay variation}

The delay variation results were plotted in Figures 6 and 7 . It is shown that for a small increase, the delay variation of the MOS decreases dramatically. For a variation of only $\pm 2 \mathrm{~ms}$ the MOS for the Foreman and Hall-monitor videos decays to a value close to poor. The video sequence Football is less affected by the delay variation for values between $\pm 2 \mathrm{~ms}$ and $\pm 8 \mathrm{~ms}$. However, for bigger variations the MOS is similar for the three video sequences. Delay variation above $\pm 8 \mathrm{~ms}$ for all the video sequences leads to a MOS close to bad (1), indicating a reject of the video from the observers. In Figure 7, we can see that for the Football sequence, the MOS does not vary for delay variations between $\pm 2 \mathrm{~ms}$ and $\pm 4 \mathrm{~ms}$.

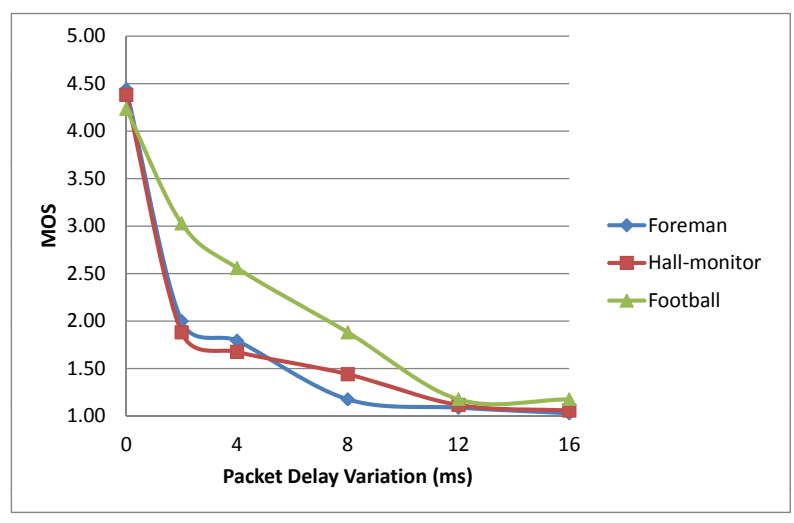

Fig. 6. MOS for delay variation displayed on the laptop

Figure 8 presents the confidence interval values for delay variation. The $\mathrm{x}$-axis shows the video sequence, the device that was used to present the video sequence to the observer and the

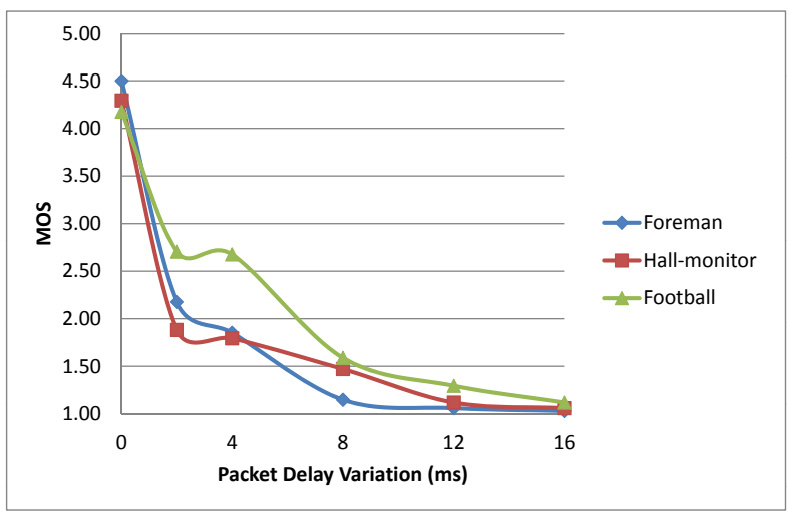

Fig. 7. MOS for delay variation displayed on the mobile phone

percentage of packet loss emulated, as explained in the context of Figure 5. The y-axis refers to the MOS. It is appreciated that the Football video sequence gets higher rates between $\pm 2 \mathrm{~ms}$ and $\pm 8 \mathrm{~ms}$, the higher rates become less obvious for the values of $\pm 12 \mathrm{~ms}$ and $\pm 16 \mathrm{~ms}$.

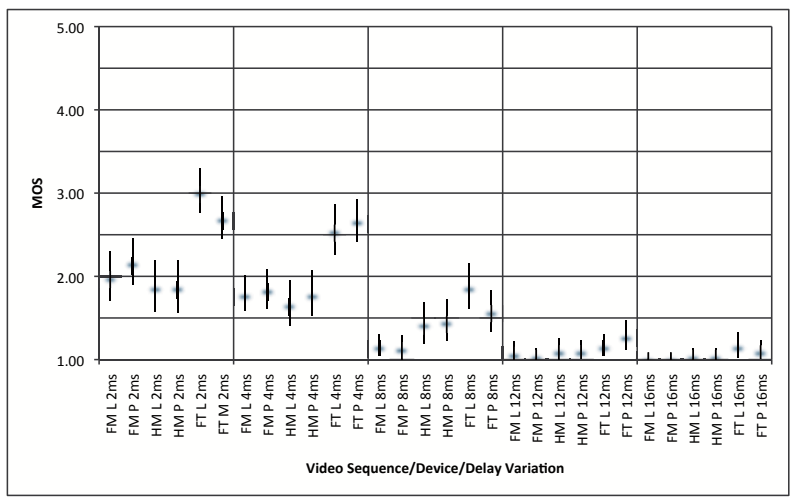

Fig. 8. 95\%-Confidence interval for delay variation

Table I shows the regression analysis of packet loss and packet delay variation shown in figures 3, 4, 6 and 7. The second-last column shows the approximation for every individual case, and the last column shows the $\mathrm{R}$ squared values that should approach one in the case of a perfect match. In the case of loss, the power regression model fitted best, while in the case of delay, an exponential function was superior. The MOS values for zero loss ratio and zero packet delay variation could not be considered for the power regression model and were thus disregarded in general.

Despite not adding both disturbances to the same video, we can notice that it is likely that the perception of quality in the video degrades more for small changes in the delay variation. This is similar to the findings of the study performed by Fiedler et al. [20] for the type-p reordered ratio in case of the iLBC codec.

\section{Mobile phone and laptop}

The MOS for the packet loss and delay variation was plotted for both devices, the laptop and mobile phone, in Figure 9 


\begin{tabular}{|c|c|c|c|c|}
\hline Parameter & Video & Device & Equation & $R^{2}$ \\
\hline \multirow{6}{*}{$\begin{array}{l}\text { Loss } \\
\text { Ratio } \\
(L)\end{array}$} & \multirow[t]{2}{*}{ Foreman } & Laptop & $\min \left(1+1.23 L^{-0.585}, 5\right)$ & 0.969 \\
\hline & & Mobile & $\min \left(1+1.40 L^{-0.802}, 5\right)$ & 0.786 \\
\hline & \multirow{2}{*}{$\begin{array}{c}\text { Hall- } \\
\text { Monitor }\end{array}$} & Laptop & $\min \left(1+1.59 L^{-0.427}, 5\right)$ & 0.960 \\
\hline & & Mobile & $\min \left(1+1.48 L^{-0.394}, 5\right)$ & 0.983 \\
\hline & \multirow[t]{2}{*}{ Football } & Laptop & $\min \left(1+1.31 L^{-0.816}, 5\right)$ & 0.851 \\
\hline & & Mobile & $\min \left(1+1.16 L^{-0.583}, 5\right)$ & 0.874 \\
\hline \multirow{6}{*}{$\begin{array}{l}\text { Delay } \\
\text { Variation } \\
(J)\end{array}$} & \multirow[t]{2}{*}{ Foreman } & Laptop & $1+1.79 \exp (-0.258 J)$ & 0.986 \\
\hline & & Mobile & $1+1.99 \exp (-0.279 J)$ & 0.971 \\
\hline & \multirow{2}{*}{$\begin{array}{c}\text { Hall } \\
\text {-Monitor }\end{array}$} & Laptop & $1+1.53 \exp (-0.201 J)$ & 0.967 \\
\hline & & Mobile & $1+1.68 \exp (-0.207 J)$ & 0.959 \\
\hline & \multirow[t]{2}{*}{ Football } & Laptop & $1+3.21 \exp (-0.197 J)$ & 0.920 \\
\hline & & Mobile & $1+3.02 \exp (-0.199 J)$ & 0.985 \\
\hline
\end{tabular}

TABLE I

MOS TRend Fitting of Packet loss and Delay Variation; $L$ is GIVEN IN \%, AND $J$ IS GIVEN IN MS.

and Figure 10, respectively. For both graphs we can notice that the results are almost the same for both devices. The biggest difference is found for packet loss of $0.4 \%$. In this case, weobtained a MOS of 3.19 for the laptop, and for the mobile phone a slightly higher rating of 3.34 .

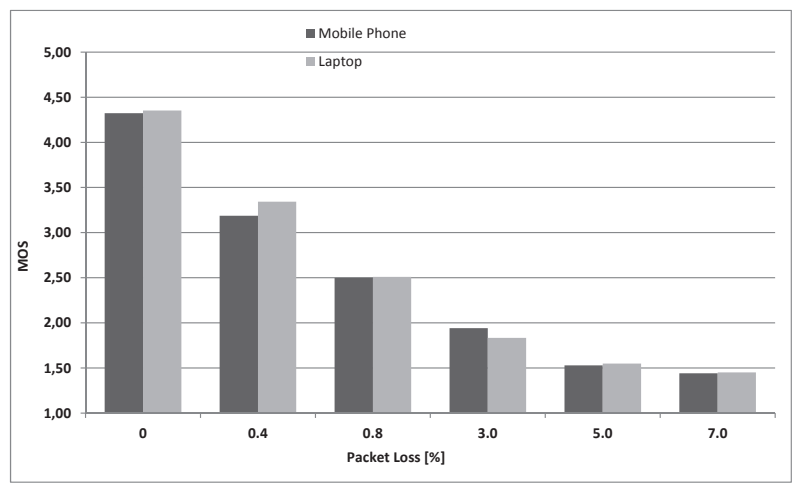

Fig. 9. MOS packet loss displayed on the laptop and mobile phone

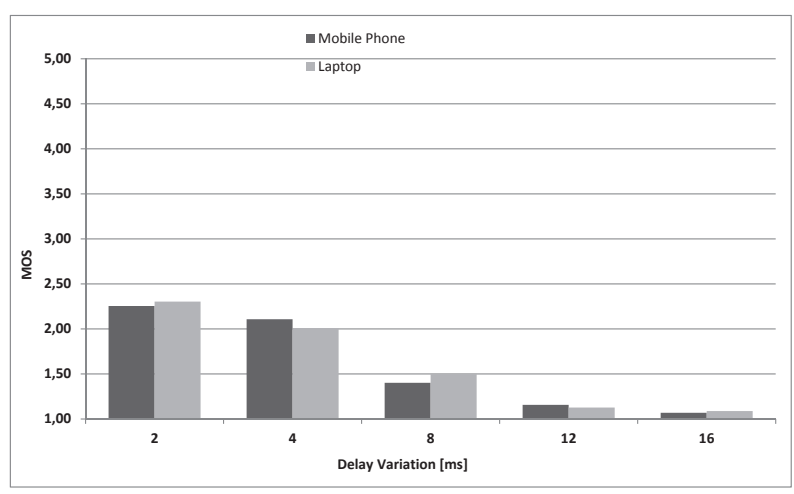

Fig. 10. MOS delay variation displayed on the laptop and mobile phone

To identify if these variations are significant, we applied a matched-sample $t$-test. This test is used when we have two matched or correlated samples, and when we need to test the difference between two averages [27]. In this specific case, we compared the mean between the ratings per user obtained for the laptop and the ones obtained when the observers used the mobile phone as displaying device.

Let us consider that $\bar{u}_{L}$ is the average for the laptop, $\bar{u}_{P}$ is the average rating for the mobile phone. Thus, the hypothesis is if $\bar{u}_{L}=\bar{u}_{P}$ is true, then there is no difference between doing the experiment on the mobile phone or on the laptop when using the same resolution in both devices.

If we consider the difference of the means $\bar{u}_{D}=\bar{u}_{L}-\bar{u}_{P}$, then the hypothesis can also be expressed as:

$$
H: \bar{u}_{D}=\bar{u}_{L}-\bar{u}_{P}=0
$$

This test requires the calculation of the $t_{\text {calc }}$ parameter, which is defined as:

$$
t_{\text {calc }}=\frac{\bar{D}-\bar{u}_{D}}{\frac{s_{D}}{\sqrt{N}}}
$$

where $\bar{D}$ and $s_{D}$ are the mean and standard deviation of the difference scores between the data obtained for the laptop and mobile phone. According to our hypothesis, $\bar{u}_{D}=0$, and $N$ is the number pairs of difference scores.

After calculating the $t_{c a l c}$ parameter, it is compared with $t_{\text {table }}$ that is obtained from the Student $t$ distribution table [27]. The $t_{\text {table }}$ value depends on the level of significance, in this case a $95 \%$ confidence interval is used. Thus, the level of significance is 0.05 , the degrees of freedom (df) is defined as $N-1=33$. The value from the table to compare in our case corresponds to $t_{\text {table }}=2.042$. If $t_{\text {calc }}<t_{\text {table }}$ we fail to reject the hypothesis, so there is no evidence to suggest that the different devices affect the perception of the shown video.

Table II presents the results for the calculations for each of the video pairs, referring to the same video sequence presented on the laptop and on the mobile phone. The comparison of $t_{\text {calc }}$ with $t_{\text {table }}$ reveals that for the most of the video sequences, there is no evidence to suggest that the different devices affect the perception of the shown video. Therefore, we can conclude that there is not a significant difference between doing the experiment on the mobile phone and on the laptop displaying the same resolution. Overall very similar rating behaviors were observed for both devices.

\section{CONClusion}

In this paper we investigated the mobile video quality of experience, encoded with the H.264 baseline profile. The network performance parameter plays an important role on the quality of streamed video. In this study we focused on the effect of packet loss and packet delay variation on mobile video quality of experience. We found that mobile video encoded with the H. 264 baseline profile is very sensitive to the network disturbance, and that user ratings dropped quickly with nominal packet loss and packet delay variation. Moreover the users rating survey is conducted on mobile phone as well as on the laptop. Results for both devices were compared using matched-sample t-test. We did not find any strong evidence to conclude that devices have any impact on user perception for video which has the same resolution. In other words, 


\begin{tabular}{|c|c|c|c|c|c|c|c|c|}
\hline \multicolumn{3}{|c|}{ Foreman } & \multicolumn{3}{|c|}{ Hall-Monitor } & \multicolumn{3}{|c|}{ Football } \\
\hline$L$ & $t_{\text {calc }}$ & Hypothesis & $L$ & $t_{\text {calc }}$ & Hypothesis & $L$ & $t_{\text {calc }}$ & Hypothesis \\
\hline $0.0 \%$ & -0.63 & Fail to reject & $0.0 \%$ & 0.90 & Fail to reject & $0.0 \%$ & 0.42 & Fail to reject \\
\hline $0.4 \%$ & -1.14 & Fail to reject & $0.4 \%$ & 2.50 & Reject & $0.4 \%$ & 3.20 & Reject \\
\hline $0.8 \%$ & 0.42 & Fail to reject & $0.8 \%$ & 1.36 & Fail to reject & $0.8 \%$ & -2.03 & Fail to reject \\
\hline $3.0 \%$ & -2.05 & Fail to reject & $3.0 \%$ & -0.44 & Fail to reject & $3.0 \%$ & 0.25 & Fail to reject \\
\hline $5.0 \%$ & -0.25 & Fail to reject & $5.0 \%$ & -0.27 & Fail to reject & $5.0 \%$ & 1.16 & Fail to reject \\
\hline $7.0 \%$ & 0.00 & Fail to reject & $7.0 \%$ & 0.83 & Fail to reject & $7.0 \%$ & -0.63 & Fail to reject \\
\hline$D \pm \Delta D$ & $t_{\text {calc }}$ & Hypothesis & $D \pm \Delta D$ & $t_{\text {calc }}$ & Hypothesis & $D \pm \Delta D$ & $t_{\text {calc }}$ & Hypothesis \\
\hline $150 \pm 2 \mathrm{~ms}$ & -1.36 & Fail to reject & $150 \pm 2 \mathrm{~ms}$ & 0.00 & Fail to reject & $150 \pm 2 \mathrm{~ms}$ & 2.46 & Reject \\
\hline $150 \pm 4 \mathrm{~ms}$ & 0.70 & Fail to reject & $150 \pm 4 \mathrm{~ms}$ & -1.28 & Fail to reject & $150 \pm 4 \mathrm{~ms}$ & -0.89 & Fail to reject \\
\hline $150 \pm 8 \mathrm{~ms}$ & 0.44 & Fail to reject & $150 \pm 8 \mathrm{~ms}$ & -0.37 & Fail to reject & $150 \pm 8 \mathrm{~ms}$ & 2.73 & Reject \\
\hline $150 \pm 12 \mathrm{~ms}$ & 0.44 & Fail to reject & $150 \pm 12 \mathrm{~ms}$ & 0.00 & Fail to reject & $150 \pm 12 \mathrm{~ms}$ & -1.68 & Fail to reject \\
\hline $150 \pm 16 \mathrm{~ms}$ & 0.00 & Fail to reject & $150 \pm 16 \mathrm{~ms}$ & 0.00 & Fail to reject & $150 \pm 16 \mathrm{~ms}$ & 0.81 & Fail to reject \\
\hline
\end{tabular}

TABLE II

MATCHED-SAMPle $t$ TeST RESUlTs, with $t_{\text {calc }}=2.042$

the assessment of mobile video quality can be performed on laptops as well; mobile phones are not needed.

\section{REFERENCES}

[1] D. Wu, Y. Hou, W. Zhu, Y.-Q. Zhang, and J. Peha, "Streaming video over the internet: Approaches and directions," IEEE Transactions on Circuits and Systems for Video Technology, vol. 11, no. 3, pp. 282-300, 2001.

[2] ITU-T REC-H.264, "H.264 : Advanced video coding for generic audiovisual services." International Telecommunications Union Telecommunication Sector Publications, March 2010. [Online]. Available: http://www.itu.int/rec/T-REC-H.264-201003-I/en

[3] L. Bouchard, "Multimedia software for mobile phones," Software, IEEE, vol. 27, no. 3, pp. $8-10$, May-June 2010.

[4] J. G. Apostolopoulos, W. tian Tan, and S. J. Wee, "Video streaming: Concepts, algorithms, and systems," HP Laboratories, 2002, Tech. Rep., 2002.

[5] ITU-T P.910, "Subjective video quality assessment methods for multimedia applications." International Telecommunications Union Telecommunication Sector, 1999.

[6] ITU-R BT.500-11, "Methodology for the subjective assessment of the quality of television pictures," International Telecommunications Union Radiocommunication Sector, 2002.

[7] P. Calyam, M. Sridharan, W. Mandrawa, and P. Schopis, "Performance measurement and analysis of H.323 traffic," in Passive and Active Network Measurement, ser. Lecture Notes in Computer Science, C. Barakat and I. Pratt, Eds. Springer Berlin / Heidelberg, 2004, vol. 3015, pp. 137-146. [Online]. Available: http://dx.doi.org/10.1007/ 978-3-540-24668-8_14

[8] M. Claypool and J. Tanner, "The effects of jitter on the peceptual quality of video," in MULTIMEDIA '99: Proceedings of the seventh ACM international conference on Multimedia (Part 2). ACM, 1999, pp. $115-118$.

[9] D. Hands and M. Wilkins, "A study of the impact of network loss and burst size on video streaming quality and acceptability," in Interactive Distributed Multimedia Systems and Telecommunication Services, M. Diaz, P. Owezarski, and P. Snac, Eds. Berlin, Heidelberg: Springer Berlin Heidelberg, 1999, vol. 1718, pp. 45-57. [Online]. Available: http://www.springerlink.com/content/21u2413r58534152/

[10] S. Jumisko-Pyykkö and J. Häkkinen, "Evaluation of subjective video quality of mobile devices," in MULTIMEDIA '05: Proceedings of the 13th annual ACM international conference on Multimedia, 2005, pp. 535-538.

[11] T.-L. Lin, S. Kanumuri, Y. Zhi, D. Poole, P. Cosman, and A. Reibman, "A versatile model for packet loss visibility and its application to packet prioritization," IEEE Transactions on Image Processing, vol. 19, no. 3, pp. $722-735,2010$.

[12] D. Loguinov and H. Radha, "Measurement study of low-bitrate internet video streaming," in Proceedings of the 1st ACM SIGCOMM Workshop on Internet Measurement, 2001, pp. 281-293. [Online]. Available: http://doi.acm.org/10.1145/505202.505238
[13] F. De Simone, M. Tagliasacchi, M. Naccari, S. Tubaro, and T. Ebrahimi, "A H.264/AVC video database for the evaluation of quality metrics," in Acoustics Speech and Signal Processing (ICASSP), 2010 IEEE International Conference on, 2010, pp. $2430-2433$.

[14] M. Pinson, S. Wolf, and G. Cermak, "HDTV Subjective quality of H.264 vs. MPEG-2, with and without packet loss," IEEE Transactions on Broadcasting, vol. 56, no. 1, pp. 86-91, 2010.

[15] M. Ries, O. Nemethova, and M. Rupp, "Video quality estimation for mobile H. 264/AVC video streaming," Journal of Communications, vol. 3, no. 1, pp. 41-50, 2008.

[16] V. Vassiliou, P. Antoniou, I. Giannakou, and A. Pitsillides, "Requirements for the transmission of streaming video in mobile wireless networks," in Artificial Neural Networks ICANN 2006, ser. Lecture Notes in Computer Science, 2006, vol. 4132, pp. 528-537. [Online]. Available: http://dx.doi.org/10.1007/11840930_55

[17] S. Winkler and F. Dufaux, "Video quality evaluation for mobile applications," in Proceedings of SPIE Conference on Visual Communications and Image Processing, vol. 5150, 2003, pp. 593-603.

[18] H. Knoche, J. D. McCarthy, and M. A. Sasse, "Can small be beautiful?: Assessing image resolution requirements for mobile TV," in MULTIMEDIA '05: Proceedings of the 13th annual ACM international conference on Multimedia, 2005, pp. 829-838.

[19] J. Shaikh, T. Minhas, P. Arlos, and M. Fiedler, "Evaluation of delay performance of traffic shapers," in Second International Workshop on Security and Communication Networks, 2010, pp. 1-8.

[20] M. Fiedler, T. Hossfeld, and P. Tran-Gia, "A generic quantitative relationship between quality of experience and quality of service," IEEE Network, vol. 24, no. 2, pp. 36-41, Apr. 2010.

[21] M. Mu, A. Mauthe, and F. Garcia, "A Utility-Based QoS model for emerging multimedia applications," in The Second International Conference on Next Generation Mobile Applications, Services and Technologies, 2008. NGMAST '08. IEEE, Sep. 2008, pp. 521-528.

[22] Z. Wang, H. R. Sheikh, and A. C. Bovik, "Objective video quality assessment," in In the handbook of video databases: Design and Applications. CRC Press, 2003, pp. 1041-1078.

[23] S. Spirou, "Packet reordering effects on the subjective quality of broadband digital television," in Consumer Electronics, 2006. ISCE '06. 2006 IEEE Tenth International Symposium on, 0-0 2006, pp. 1 -6.

[24] OPTICOM, "Perceptual evaluation of video quality," [Online] http://www.pevq.org/, accessed July 2010.

[25] T. N. Minhas and M. Fiedler, "Impact of disturbance locations on video quality of experience," in Quality of Experience for Multimedia Content Sharing, EuroITV2011, June 2011.

[26] "Xiph.org test media," [Online] http://media.xiph.org/video/derf/, accessed on July 2010.

[27] D. C. Howell, Statistical Methods for Psychology, 7th ed. Wadsworth, 2010. 